\title{
Anomaly-Based JPEG2000 Compression of Hyperspectal Imagery
}

\author{
Qian Du, Senior Member, IEEE, Wei Zhu, Student Member, IEEE, and James E. Fowler, Senior Member, IEEE
}

\begin{abstract}
Lossy compression of hyperspectral imagery is considered with special emphasis on the preservation of anomalous pixels. In the proposed scheme, anomalous pixels are extracted before compression and replaced with interpolation from surrounding, non-anomalous pixels. The image is then coded using principal component analysis for spectral decorrelation followed by JPEG2000. The anomalous pixels do not participate in this lossy compression and are rather transmitted separately in a lossless fashion. Upon decoding, the anomalous pixels are inserted back into the image. Experimental results demonstrate that the proposed scheme improves not only anomaly detection performed subsequent to decoding but also the rate-distortion performance of the lossy-compression process.
\end{abstract}

Index Terms-Hyperspectral compression, anomaly detection, principal component analysis

\section{INTRODUCTION}

In hyperspectral image analysis, anomalies are important pixels since they often represent man-made targets of critical application interest. Detection of anomalies is very useful in some critical situations, such as in applications related to national defense and law enforcement. During lossy compression, it is therefore essential to preserve these pixels in order to enable target detection and discrimination in an unsupervised setting when data storage and transmission capabilities are limited (e.g., small airborne platforms). However, anomalous pixels are difficult to compress well due to the fact that their spectral features are typically quite different from their surroundings. Indeed, anomalous pixels are often among those that experience the largest distortion after lossy compression. This phenomenon is due to the fact that most lossy compression algorithms are designed-either explicitly or implicitly - to minimize some reconstruction-distortion criterion that is typically expressed as a global average across the entire dataset. For example, many compression algorithms are designed inherently to minimize a mean squared error (MSE) between the original and reconstructed images. Other distortion measures, such as signal-to-noise ratio (SNR) or an average spectral angle, also gauge quality in a global sense as averaged over the entire dataset. Because they account for only a relatively small portion of the dataset, anomalous pixels do not tend to contribute significantly to such average measures; as a consequence, anomalous pixels may be severely distorted in the compression process. Therefore, special care should be taken to preserve anomalous pixels.

In [1], a process was proposed to adjust anomalies prior to compression in order to enhance their reconstruction quality.

The authors are with the Department of Electrical \& Computer Engineering, Mississippi State University, Starkville, MS, USA, and the GeoResources Institute (GRI) at the Mississippi State High Performance Computing Collaboratory $\left(\mathrm{HPC}^{2}\right)$.
In this technique, it is attempted to make anomalies look more like their surroundings to facilitate their compression; upon decompression, the adjustment is inverted to restore the anomalous character to the adjusted pixels. In this paper, we propose an alternative in which, prior to compression, anomalous pixels are completely removed from the dataset and replaced by an interpolation amenable to efficient compression. The anomalies are transmitted separately such that they can be restored to the dataset upon decompression. As compared to the technique of [1], our proposed approach improves both the rate-distortion performance of the lossy compression as well as the performance of anomaly detection applied subsequent to dataset reconstruction in situations in which the anomaly model of [1] fails.

The remainder of this paper is organized as follows. In the next section, we briefly overview the popular approach to hyperspectral compression that consists of JPEG2000 [2,3] coupled with spectral decorrelation via principal component analysis (PCA) since both our proposed approach as well as that of [1] use such a coder as the core compression engine. Next, in Sec. III, we overview the technique of adjusting anomalies for compression as proposed in [1]. We then outline our proposed approach-anomaly removal-in Sec. IV. Experimental results in Sec. V compare our proposed anomalyremoved approach to the anomaly-adjusted approach of [1]. Finally, we make some concluding comments in Sec. VI.

\section{JPEG2000 AND PCA}

It has been shown [4-6] that PCA in conjunction with JPEG2000 [2] can provide superior rate-distortion performance for hyperspectral image compression, where PCA provides spectral decorrelation prior to the application of JPEG2000 to the resulting principal component (PC) images (we refer to this as "PCA+JPEG2000"). In particular, PCA+JPEG2000 outperforms DWT+JPEG2000, the corresponding strategy that uses a discrete wavelet transform (DWT) for spectral decorrelation. In this sense, spectral decorrelation is critical for hyperspectral compression, and PCA outperforms the DWT in this respect. When only the most significant PCs are retained and compressed, the resulting algorithm, denoted as SubPCA+JPEG2000, can yield even better performance than PCA+JPEG2000, particularly at low bitrates $[7,8]$. For example, Table I provides rate-distortion performance-including those for two other common DWTbased algorithms, 3D-SPIHT [9] and 3D-SPECK [10]-for a popular AVIRIS dataset, Jasper Ridge. Both PCA-based algorithms clearly outperform the group of DWT-based schemes by a wide margin. As for spectral fidelity, one can employ 
the spectral angle mapper (SAM) and compute the average of spectral angles between the original and reconstructed pixels. As tabulated in Table I, the PCA-based algorithms also provide reconstructed data with higher spectral fidelity by this measure. As a consequence, we focus on PCA+JPEG2000 and SubPCA+JPEG2000 from this point on. We note that both techniques use the multicomponent transform (MCT) extension permitted by Part 2 [3] of the JPEG2000 standard.

\section{Anomaly-Adjusted COMPREssion}

In [1], a procedure for preserving anomalous pixels in compression was proposed. In this scheme, anomalous pixels are detected using the well-known RX algorithm [11,12]. After applying PCA-based spectral decorrelation, the identified anomalous pixels are adjusted by mean-removal; i.e., the anomalous pixels are averaged, and this resulting mean is subtracted from each anomalous pixel. Afterwards, JPEG2000 is applied to the entire image. Upon decoding, the anomalouspixel mean, losslessly transmitted separately, is restored to each of the anomalous pixels. As a result, the spectral fidelity of the anomalous pixels is improved upon what would have resulted from direct application of the lossy compression to all pixels without adjustment of the anomalies. Consequently, the performance of anomaly detection subsequent to reconstruction is improved, while the rate-distortion performance of the lossy compression is nearly unchanged. Below, we refer to this anomaly-adjustment (AA) procedure as "PCA+AA+JPEG2000."

We note that, in this AA approach, mean removal occurs after PCA; that is, the determination of the PCA transform matrix (typically computed by an eigendecomposition of the covariance matrix of the dataset) includes the anomalous pixels. This implies that the PCA transform will not represent the non-anomalous pixels as well as it would if the anomalies were not included in the PCA training process.

The AA approach of [1] is premised on the assumption that, although different from their surrounding pixels, the anomalies are themselves rather similar. That is, the anomaly pixels belong to a single class that shares the same statistics, specifically, the same mean vector. As we will see below, depending on the dataset, sometimes this assumption holds, but sometimes it does not. In such latter cases, the AA approach has difficulty preserving the anomaly pixels.

\section{AnOmaly-Removed Compression}

In contrast to the AA technique of [1], we propose to completely remove anomalies prior to compression. In our anomaly-removal (AR) approach, anomaly detection, such as the RX algorithm [11,12], is applied first to identify anomalous pixels. These pixels are then extracted from the dataset and transmitted (losslessly) independently of the remainder of the dataset. In order to compress the rest of the image, the anomalous pixels in the original dataset are replaced by values interpolated from neighboring pixels. Specifically, for an isolated anomalous pixel vector, the anomaly is replaced with the average of the eight pixels immediately surrounding it spatially. For larger regions, the entire anomalous region is replaced by the average of the non-anomalous pixels calculated along the boundary of the region. Since this spatial-averaging interpolation is a simple form of lowpass filtering, high spatialfrequency components produced by anomalous pixels tend to be suppressed, leading to increased compression efficiency. The PCA spectral transform is then calculated and applied to the resulting dataset, followed by either PCA+JPEG2000 or SubPCA+JPEG2000 coding. After decoding, the original anomalous pixels are inserted back into the reconstructed image. Below, we refer to the resulting algorithms as "AR+PCA+JPEG2000" or "AR+SubPCA+JPEG2000," respectively, depending on whether the full complement of PCs generated by spectral PCA is coded or not.

In order to permit restoration of the anomalous pixels after decompression, several items of ancillary data are required to be provided by the encoder separate from the JPEG2000 compressed bitstream. Specifically, each anomalous pixel vector must be specified, as well as its location within the dataset. The amount of ancillary data will vary depending on the spatial size of the dataset as well as the number of spectral bands and the number of anomalies identified. In experiments below, we represent each anomalous pixel vector (uncompressed) using 16 bits per vector component; for anomaly locations, we represent the row and column indices using 9 bits each. We note that, although this ancillary information is technically independent from the JPEG2000 bitstream, it can be embedded directly into a JP2- or JPX-format compressed file with one or more UUID blocks [2] which are designed to carry applicationspecific user data. Finally, we note that the AA approach of [1] also requires similar ancillary data—in addition to anomaly locations, merely a single mean vector is sent. Even though our AR approach requires more ancillary data, in both cases, the amount of overhead is typically much smaller than that associated with the MCT marker segment [3] used to transmit the PCA transform matrix within the JPEG2000 bitstream.

\section{EXPERIMENTAL RESULTS}

In the following experiments, we employ SNR for data fidelity since it is widely used for assessment of rate-distortion performance and has been employed in our previous studies; a group of alternative quality measures for hyperspectral imagery can be found in [13]. Additionally, we conduct post-compression anomaly detection as an application-oriented quality assessment. Note that, for both AA and AR, anomalydetection results can be retrieved directly from the compressed bitstream since the anomaly locations are transmitted losslessly. The post-compression anomaly detection conducted here (and in [1]) is intended simply as a means to objectively evaluate how well anomalies can be extracted from the reconstructed image. We will see that, even in the case that anomalies are perfectly preserved, some anomalies may fail to be detected while some background pixels may produce false alarms due to compression effects on the background.

\section{A. AVIRIS Results}

Fig. 1 depicts Moffett, an AVIRIS image of size $512 \times$ 512 with 224 spectral bands. Fig. 2 shows the corresponding anomaly-detection map resulting from the RX algorithm 
wherein 59 anomalies are identified, accounting for $0.02 \%$ of the total pixels. Here, we have implemented a global RX anomaly detector as described in [12]. Below, all bitrate figures include appropriate overhead information for the anomalies and their locations.

Fig. 3 and Table II present rate-distortion performance of the six algorithms we consider here. ${ }^{1}$ We see that all SubPCA variants provide larger SNR values than their PCA counterparts, particularly at the lower bitrates. Additionally, the AA and AR variants perform essentially the same as the original PCA+JPEG2000 and SubPCA+JPEG2000, indicating that the anomalies have very little effect on the rate-distortion performance for this dataset. In fact, we see less than a 0.1-dB difference between the AR and AA coders and between these coders and those that do not handle anomalies specially.

Fig. 4 shows the receiver operating characteristic (ROC) curves for the six algorithms. This ROC performance plots probability of detection, $P_{d}$, versus probability of false alarm, $P_{f}$, associated with anomaly detection performed on the reconstructed data after compression at 1.0 bpppb. $^{2}$ We see that PCA+AA+JPEG2000 slightly outperforms PCA+JPEG2000, while both are outperformed by SubPCA+JPEG2000, SubPCA+AA+JPEG2000, AR+PCA+JPEG2000, and AR+SubPCA+JPEG2000 which all perform roughly equivalently.

\section{B. CASI Results}

Fig. 5 depicts CASI data of size $150 \times 250$ with 72 bands. This dataset, having spatial resolution about $8 \mathrm{~nm}$ and spatial resolution about $2 \mathrm{~m}$, is a scene surrounding a residential community close to Mississippi State University taken in May 2002. Fig. 6 depicts the corresponding anomaly-detection map resulting from the $\mathrm{RX}$ algorithm wherein 16 anomalies are identified, accounting for $0.04 \%$ of the total pixels.

In this case, the AR algorithms significantly outperform the other techniques in rate-distortion performance as clearly seen in Fig. 7 and Table III. AR+SubPCA+JPEG2000 is the top-performing approach, outperforming both SubPCA+AA+JPEG2000 as well as SubPCA+JPEG2000, which in turn do better than their PCA counterparts. Of particular note is that, at $1.0 \mathrm{bpppb}$, the proposed AR variants yield about a 5-dB gain over the corresponding AA techniques.

Fig. 8 presents the ROC curves from the six algorithms resulting from anomaly detection on the reconstructed data after compression at $1.0 \mathrm{bpppb}^{3}$ In this case, we observe that PCA+AA+JPEG2000 outperforms SubPCA+AA+JPEG2000; similarly, PCA+JPEG2000 outperforms SubPCA+JPEG2000. However, AR+PCA+JPEG2000 and AR+SubPCA+JPEG2000 both perform roughly equivalently. Most prominently though

\footnotetext{
${ }^{1}$ Note that only PCA+AA+JPEG2000 was originally considered in [1] however, SubPCA+AA+JPEG2000 is easily obtained by simply discarding minor PCs before encoding. Also note that PCA+AA+JPEG2000, PCA+JPEG2000, SubPCA+AA+JPEG2000, and SubPCA+JPEG2000 all use the same PCA transform matrix.

${ }^{2}$ For SubPCA, we retain 104 out of the original 224 PCs for compression at $1.0 \mathrm{bpppb}$.

${ }^{3}$ For SubPCA, we retain 49 out of the original 72 PCs for compression at $1.0 \mathrm{bpppb}$.
}

is that the AA techniques perform almost the same as the nonanomaly-based techniques while the AR approaches decidedly achieve the best performance.

\section{Discussion}

The results above indicate a significant difference in performance between the two datasets. The root of this difference can be observed in Table IV which considers two statistics for the anomalous pixels - the angle $\left(\theta_{1}\right)$ between an individual anomaly and the global mean pixel vector, and the angle $\left(\theta_{2}\right)$ between an individual anomaly and the mean pixel vector of all the anomalies. Table IV succinctly demonstrates that the fundamental premise underlying the AA approach-that anomalies belong to a single pixel class well-represented by a mean vector-largely holds for the Moffett image since the average $\theta_{2}$ is relatively small. As a result, for the Moffett image, the AA approach is an effective model for anomaly representation, as was observed in the results of Sec. V-A. Additionally, the anomaly pixel class itself does not differ to a large extent from the non-anomalous pixels, $\theta_{1}$ being rather small. Consequently, the anomalies have little impact on the rate-distortion performance of lossy compression.

On the other hand, we see from Table IV that, in the CASI dataset, anomalies differ rather significantly from both the non-anomalous pixels (large $\theta_{1}$ ) as well as from other anomalies (large $\theta_{2}$ ). In this case, AA is largely ineffective at preserving the anomalies - as seen in the results of Sec. V-B, PCA+AA+JPEG2000 is no better than PCA+JPEG2000 in either rate-distortion performance or post-compression anomaly detection. On the other hand, because the AR techniques preserve the anomalies exactly, there is a significant improvement in not only rate-distortion performance but also postcompression anomaly detection.

For the CASI dataset, we see that the PCA approaches outperform the SubPCA approaches at post-compression anomaly detection for both the AA and non-anomaly coders. This indicates that, for this dataset with a relatively small number of spectral bands (PCA dimensionality), substantial information concerning the anomalies is present in the minor, insignificant PCs that are discarded in forming SubPCA. The Moffett dataset does not manifest this phenomenon since the major PCs apparently suffice to represent well both the classes of anomalous and non-anomalous pixels. Additionally, this phenomenon does not pose an issue for the proposed AR variants since the anomalies are removed from the dataset before the PCA transform is determined or applied. In this case, AR+SubPCA+JPEG2000 performs roughly equivalently to $\mathrm{AR}+\mathrm{PCA}+\mathrm{JPEG} 2000$.

The proposed AR algorithm has computational complexity similar to that of previous algorithms such as PCA+JPEG2000 since the detected anomalies are transmitted uncompressed and the computation required for interpolation is relatively negligible. Although anomaly detection itself involves some non-negligible computation, it is assumed that this is a cost that must ultimately be borne somewhere in the system to satisfy application needs. 


\section{CONCLUSIONS}

In this paper, we proposed a process wherein anomalies are completely removed from a hyperspectral dataset and replaced by simple interpolation prior to lossy compression based on PCA spectral decorrelation and JPEG2000 coding. Upon decoding, the anomalies, which are transmitted along with their locations in a lossless fashion separately from the compressed bitstream, are restored to the reconstructed image. This proposed anomaly removal has the potential to significantly outperform a corresponding technique in [1] in which anomalies are adjusted by a mean-removal process to make them appear less different from their surroundings. The key difference between our technique and that of [1] is that the anomaly adjustment of [1] is inherently based on the assumption that anomalies belong to a single pixel class that shares the same statistics, specifically the same mean. In contrast, our anomaly removal makes no such assumption, and, instead, preserves all anomalies exactly intact. Experimental results reveal that, depending whether the single-anomalyclass assumption holds for a specific dataset or not, anomaly removal can significantly outperform the anomaly adjustment of [1] in not only rate-distortion performance, but also performance in post-compression anomaly detection.

\section{REFERENCES}

[1] B. Penna, T. Tillo, E. Magli, and G. Olmo, "Hyperspectral image compression employing a model of anomalous pixels," IEEE Geoscience and Remote Sensing Letters, vol. 4, no. 4, pp. 664-668, October 2007.

[2] Information Technology-JPEG 2000 Image Coding System-Part 1: Core Coding System, ISO/IEC 15444-1, 2000.

[3] Information Technology-JPEG 2000 Image Coding System-Part 2: Extensions, ISO/IEC 15444-2, 2004.

[4] J. E. Fowler and J. T. Rucker, "3D wavelet-based compression of hyperspectral imagery," in Hyperspectral Data Exploitation: Theory and Applications, C.-I. Chang, Ed. Hoboken, NJ: John Wiley \& Sons, Inc., 2007, ch. 14, pp. 379-407.

[5] B. Penna, T. Tillo, E. Magli, and G. Olmo, "Progressive 3-D coding of hyperspectral images based on JPEG 2000," IEEE Geoscience and Remote Sensing Letters, vol. 3, no. 1, pp. 125-129, January 2006.

[6] — , "Transform coding techniques for lossy hyperspectral data compression," IEEE Transactions on Geoscience and Remote Sensing, vol. 45, no. 5, pp. 1408-1421, May 2007.

[7] Q. Du and J. E. Fowler, "Hyperspectral image compression using JPEG2000 and principal component analysis," IEEE Geoscience and Remote Sensing Letters, vol. 4, no. 2, pp. 201-205, April 2007.

[8] W. Zhu, "On the performance of JPEG2000 and principal component analysis in hyperspectral image compression," Master's thesis, Mississippi State University, 2007.

[9] B.-J. Kim, Z. Xiong, and W. A. Pearlman, "Low bit-rate scalable video coding with 3-D set partitioning in hierarchical trees (3-D SPIHT)," IEEE Transactions on Circuits and Systems for Video Technology, vol. 10, no. 8, pp. 1374-1387, December 2000.

[10] X. Tang and W. A. Pearlman, "Scalable hyperspectral image coding," in Proceedings of the International Conference on Acoustics, Speech, and Signal Processing, vol. 2, Philadelphia, PA, March 2005, pp. 401-404.

[11] I. S. Reed and X. Yu, "Adaptive multiple-band CFAR detection of an optical patterm with unknown spectral distribution," IEEE Transactions on Acoustics, Speech, and Signal Processing, vol. 38, no. 10, pp. 1760 1770, October 1990.

[12] C.-I. Chang and S.-S. Chiang, "Anomaly detection and classification for hyperspectral imagery," IEEE Transactions on Geoscience and Remote Sensing, vol. 40, no. 6, pp. 1314-1325, June 2002.

[13] E. Christophe, D. Léger, and C. Mailhes, "Quality criteria benchmark for hyperspectral imagery," IEEE Transactions on Geoscience and Remote Sensing, vol. 43, no. 9, pp. 2103-2114, September 2005.
TABLE I

RATE-Distortion AND SPECTRAL-Fidelity PERFormanCE

\begin{tabular}{|c|c|c|c|}
\multicolumn{4}{c}{ Rate (bpppb) } \\
\cline { 2 - 4 } \multicolumn{1}{c|}{} & 0.1 & 0.5 & 1.0 \\
\hline \multirow{2}{*}{ SubPCA+JPEG2000 } & $38.55 \mathrm{~dB}$ & $46.69 \mathrm{~dB}$ & $50.41 \mathrm{~dB}$ \\
& $0.51^{\circ}$ & $0.21^{\circ}$ & $0.14^{\circ}$ \\
\hline \multirow{2}{*}{ PCA+JPEG2000 } & $36.81 \mathrm{~dB}$ & $46.53 \mathrm{~dB}$ & $50.33 \mathrm{~dB}$ \\
& $0.62^{\circ}$ & $0.21^{\circ}$ & $0.14^{\circ}$ \\
\hline \multirow{2}{*}{ DWT+JPEG2000 } & $29.00 \mathrm{~dB}$ & $39.31 \mathrm{~dB}$ & $44.85 \mathrm{~dB}$ \\
& $1.47^{\circ}$ & $0.48^{\circ}$ & $0.26^{\circ}$ \\
\hline \multirow{2}{*}{ 3D-SPECK } & $28.57 \mathrm{~dB}$ & $38.88 \mathrm{~dB}$ & $44.46 \mathrm{~dB}$ \\
& $1.56^{\circ}$ & $0.50^{\circ}$ & $0.27^{\circ}$ \\
\hline \multirow{2}{*}{ 3D-SPIHT } & $28.54 \mathrm{~dB}$ & $38.96 \mathrm{~dB}$ & $44.62 \mathrm{~dB}$ \\
& $1.56^{\circ}$ & $0.50^{\circ}$ & $0.26^{\circ}$ \\
\hline
\end{tabular}

Notes:

- Dataset is the AVIRIS Jasper Ridge radiance data of size $512 \times 512$ with 224 spectral bands.

- 3D-SPIHT and 3D-SPECK both use DWT-based spectral decorrelation.

- Distortion is measured as a signal-to-noise ratio (SNR) defined as the log ratio of signal variance to MSE

- Rate is measured in terms of bits per pixel per band (bpppb).

- Spectral fidelity is the average spectral angle in degrees between the original and reconstructed pixels.

- Results are from [8].

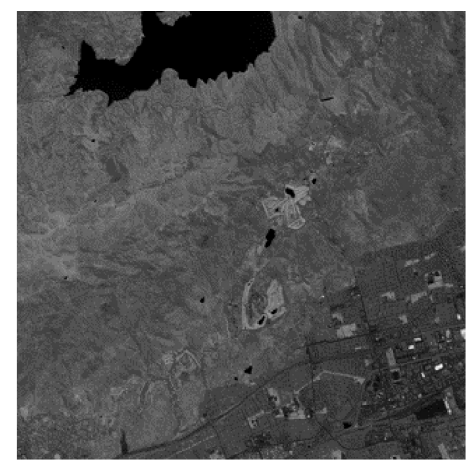

Fig. 1. Moffett, band 100.

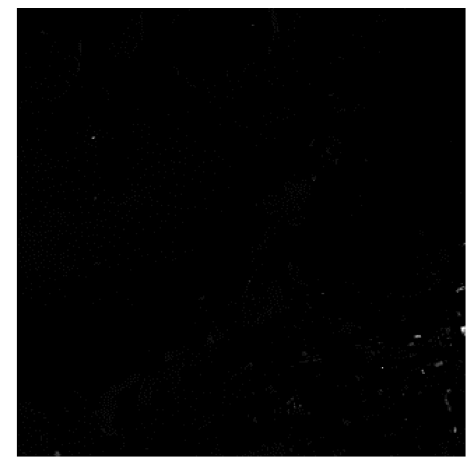

Fig. 2. Anomaly-detection map for Moffett.

TABLE II

SNR (IN DB) AT VARIOUS BITRATES FOR MOFFETT

\begin{tabular}{|c|c|c|c|}
\multicolumn{1}{c}{} & \multicolumn{3}{c}{ Rate $($ bpppb $)$} \\
\cline { 2 - 4 } Algorithm & 0.1 & 0.5 & 1.0 \\
\hline PCA+JPEG2000 & 37.49 & 47.02 & 50.92 \\
PCA+AA+JPEG2000 & 37.40 & 46.98 & 50.82 \\
AR+PCA+JPEG2000 & 37.50 & 47.04 & 50.94 \\
\hline SubPCA+JPEG2000 & 39.26 & 47.17 & 50.99 \\
SubPCA+AA+JPEG2000 & 39.20 & 47.13 & 50.90 \\
AR+SubPCA+JPEG2000 & 39.27 & 47.20 & 51.02 \\
\hline
\end{tabular}




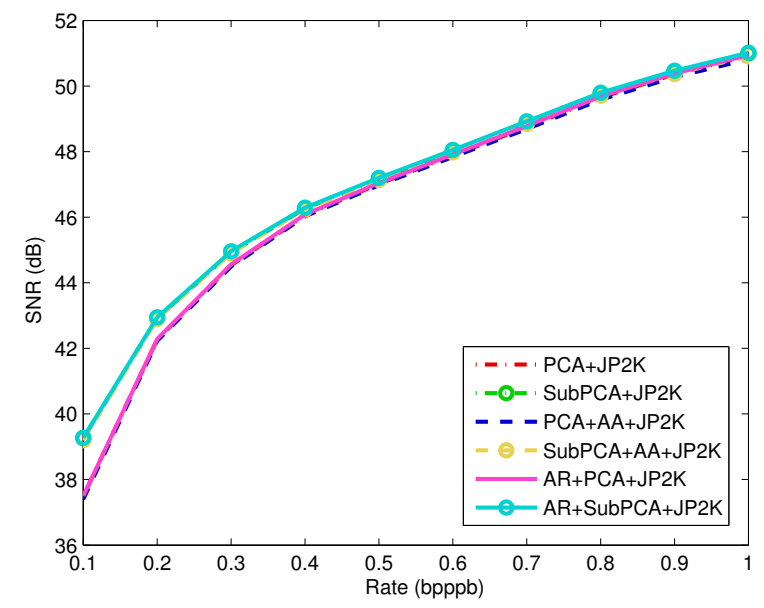

Fig. 3. Rate-distortion performance for Moffett.

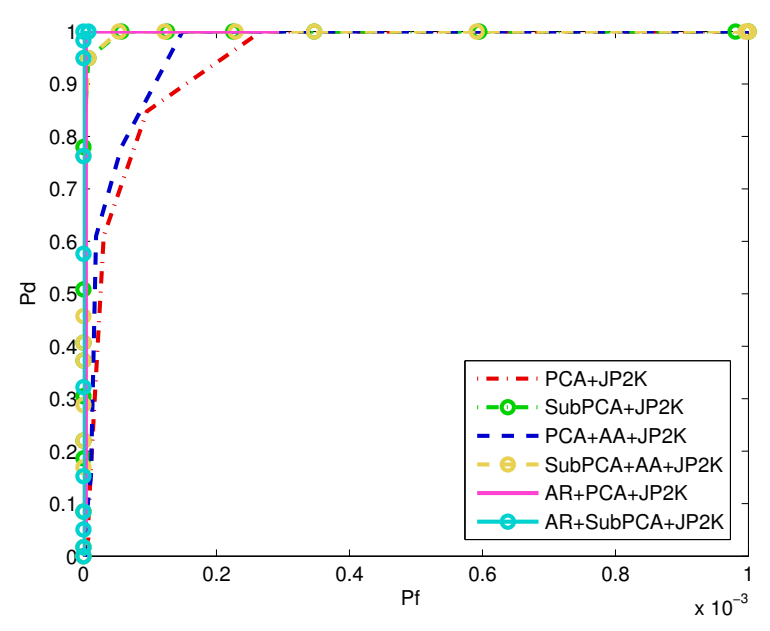

Fig. 4. ROC curves for anomaly detection performed on Moffett after compression and reconstruction at $1.0 \mathrm{bpppb}$.

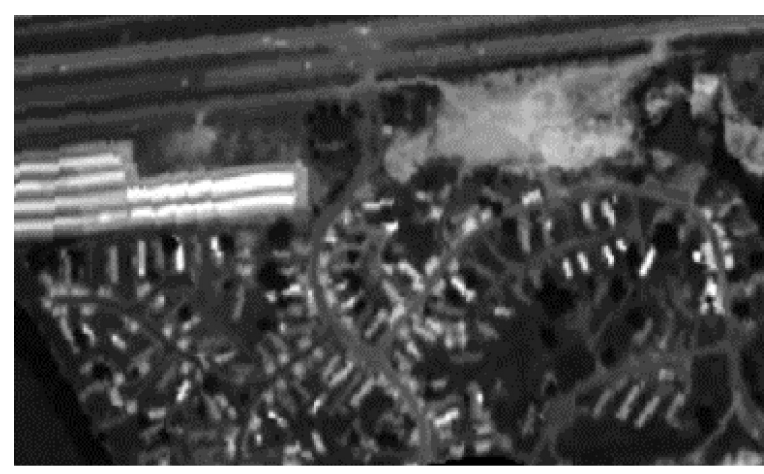

Fig. 5. The CASI dataset, band 30 .

TABLE III

SNR (IN DB) AT VARIOUS BITRATES FOR THE CASI DATASET

\begin{tabular}{|c|c|c|c|}
\multicolumn{1}{c}{} & \multicolumn{3}{c}{ Rate (bpppb) } \\
\cline { 2 - 4 } Algorithm & 0.1 & 0.5 & 1.0 \\
\hline PCA+JPEG2000 & 14.71 & 27.62 & 30.02 \\
PCA+AA+JPEG2000 & 14.60 & 27.61 & 30.02 \\
AR+PCA+JPEG2000 & 15.29 & 30.14 & 35.20 \\
\hline SubPCA+JPEG2000 & 18.65 & 27.84 & 30.05 \\
SubPCA+AA+JPEG2000 & 18.59 & 27.83 & 30.05 \\
AR+SubPCA+JPEG2000 & 19.52 & 30.57 & 35.34 \\
\hline
\end{tabular}

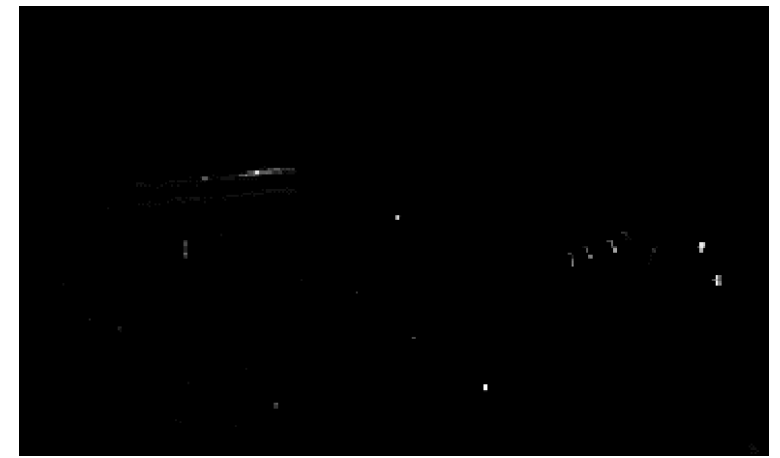

Fig. 6. Anomaly-detection map for the CASI dataset.

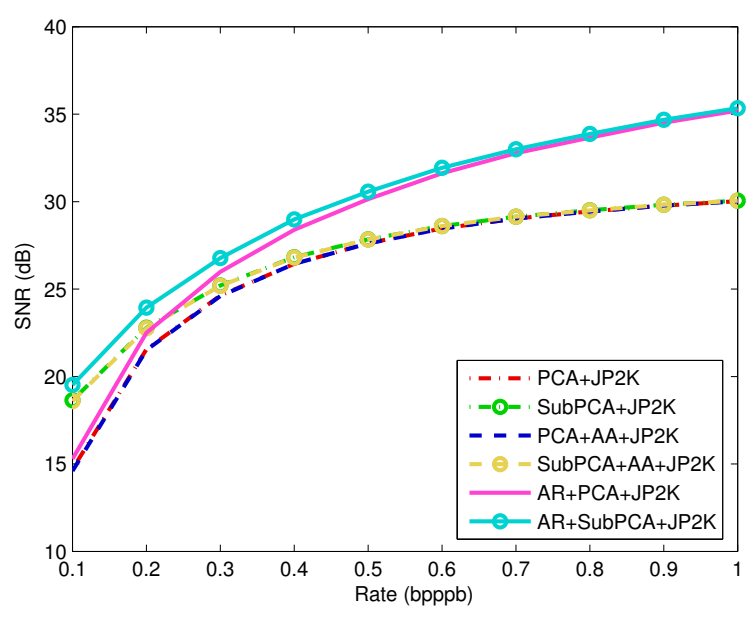

Fig. 7. Rate-distortion performance for the CASI dataset.

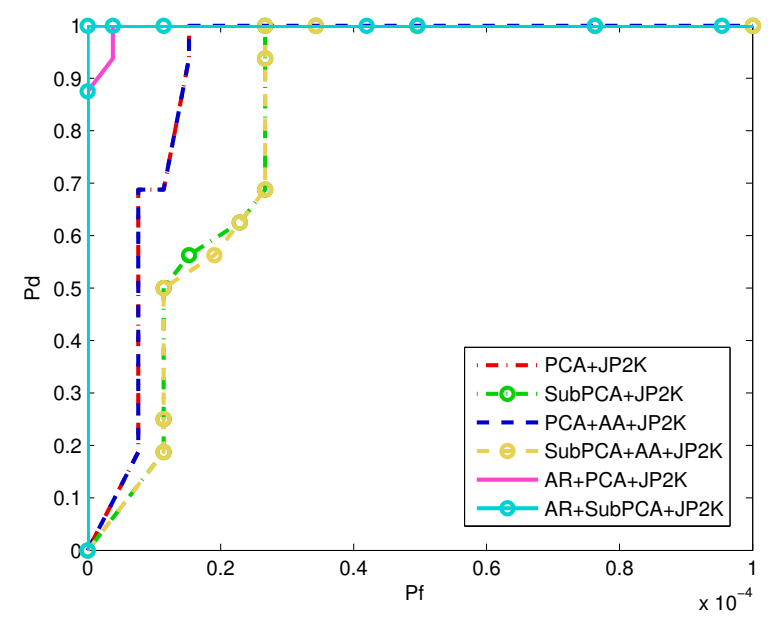

Fig. 8. ROC curves for anomaly detection performed on the CASI dataset after compression and reconstruction at $1.0 \mathrm{bpppb}$.

TABLE IV

SPeCtral Similarity BetweEn ANOMALIES AND the Other Non-ANOMALOUS PIXELS

\begin{tabular}{|c|ccc|ccc|}
\cline { 2 - 7 } \multicolumn{1}{c|}{} & \multicolumn{3}{c|}{$\theta_{1}$} & & \multicolumn{3}{c|}{$\theta_{2}$} \\
\hline Moffett & $35.8^{\circ}$ & $8.9^{\circ}$ & $15.2^{\circ}$ & $31.4^{\circ}$ & $0.9^{\circ}$ & $4.5^{\circ}$ \\
CASI & $86.0^{\circ}$ & $46.2^{\circ}$ & $70.4^{\circ}$ & $55.8^{\circ}$ & $34.4^{\circ}$ & $42.5^{\circ}$ \\
\hline
\end{tabular}

$\theta_{1}$ : angle between an individual anomaly and the global mean pixel vector $\theta_{2}$ : angle between an individual anomaly and the mean of all anomalies 